STUDI

FRANCESI

\section{Studi Francesi}

Rivista quadrimestrale fondata da Franco Simone

166 (I | LVI) | 2012

Varia

\title{
Wolfgang Drost, En route vers l'abstraction. Vibratisme vital et colorisme transcendantal: Stendhal - Baudelaire - Kandinsky
}

\section{Mario Richter}

\section{(2) OpenEdition}

\section{Journals}

\section{Edizione digitale}

URL: https://journals.openedition.org/studifrancesi/4751

DOI: 10.4000/studifrancesi.4751

ISSN: 2421-5856

\section{Editore}

Rosenberg \& Sellier

\section{Edizione cartacea}

Data di pubblicazione: 1 avril 2012

Paginazione: 171-172

ISSN: 0039-2944

\section{Notizia bibliografica digitale}

Mario Richter, «Wolfgang Drost, En route vers l'abstraction. Vibratisme vital et colorisme transcendantal: Stendhal - Baudelaire - Kandinsky», Studi Francesi [Online], 166 (I | LVI) | 2012, online dal 30 novembre 2015, consultato il 19 novembre 2021. URL: http://journals.openedition.org/studifrancesi/4751 ; DOI: https://doi.org/10.4000/studifrancesi.4751

Questo documento è stato generato automaticamente il 19 novembre 2021.

\section{cc) (†) $\odot$}

Studi Francesi è distribuita con Licenza Creative Commons Attribuzione - Non commerciale - Non opere derivate 4.0 Internazionale. 


\title{
Wolfgang Drost, En route vers l'abstraction. Vibratisme vital et colorisme transcendantal: Stendhal - Baudelaire - Kandinsky
}

\author{
Mario Richter
}

\section{NOTIZIA}

WOLFGANG DROST, En route vers l'abstraction. Vibratisme vital et colorisme transcendantal: Stendhal - Baudelaire - Kandinsky, in AA. vV., Du Romantisme à l'Art Déco, lectures croisées, sous la direction de Rossella FROISSART, Laurent HOUSSAIS, Jean-François LUNEAU, Mélanges offerts à Jean-Paul Bouillon, Rennes, Presses Universitaires de Rennes, 2011, pp. 33-44.

1 Wolfgang Drost, noto e apprezzato conoscitore della critica d'arte dell'Ottocento, ci fa percorrere in queste pagine un suggestivo itinerario attraverso una serie di concezioni estetiche che consentono di capire per quali vie si sia progressivamente giunti all'arte non figurativa e alle sue molteplici implicazioni.

2 Il punto di partenza è Stendhal, che conferisce all'arte un impulso sensuale, un'emozione soggettiva capace di suscitare un piacere simile a quello che possono dare l'amore e la musica. Baudelaire, che gli è prossimo nella convinzione che la pittura eserciti un effetto musicale sull'osservatore, si accosta agli studi propriamente scientifici dell'epoca (Lavoisier) e alle sperimentazioni degli stessi pittori quando valuta con particolare sensibilità e acume le vibrazioni luminose nell'arte e nella natura. Per questa via si va affermando una nuova concezione del mondo, visto non più come un insieme stabile ma come una realtà in costante mutamento, palpitante, fatta di vibrazioni colorate. Drost segue con esempi di sicuro interesse il rapporto esistente all'epoca fra gli artisti e critici d'arte e gli studiosi di ottica. Così, nel momento in cui 
Auguste Laugel (L'Optique et les arts, 1869) afferma che «la sensation est l'œuvre, non du nerf optique, mais du cerveau», si assiste a un decisivo mutamento di concezione, perché il mondo esterno non è più osservato passivamente ma tramite un'attiva cooperazione mentale. Se gli impressionisti rigorosi si attenevano a una sorta di materialismo ottico con l'intento di ritrarre con fedeltà ciò che si offriva al loro occhio in un dato momento, Baudelaire, poi seguito da Gauguin e Maurice Denis, apre la strada alla ricostruzione mentale del mondo visibile operata dall'artista. Questa forma di "surnaturalisme" non si distanzia dalla ricerca scientifica del secondo Ottocento, come almeno provano le importanti sperimentazioni di Hermann von Helmholtz. Su questa via, inizialmente aperta da Stendhal e soprattutto da Baudelaire, ci s'incammina verso una progressiva svalorizzazione del mondo materiale e si giunge all'astrazionefondata su effetti di natura musicale e sulla portata emozionale del colore - della quale trattò Kandinsky nel suo celebre saggio del 1911 Du spirituel dans l'art et dans la peinture en particulier. 